\title{
Pregnancy outcomes in women with primary hyperparathyroidism
}

\author{
Ali Abood and Peter Vestergaard ${ }^{1,2}$ \\ Faculty of Medicine, Aarhus University, Aarhus, Denmark, ${ }^{1}$ Department of Endocrinology, Aalborg University \\ Hospital, Aalborg, Denmark and ${ }^{2}$ Clinical Institute, Aalborg University, Aalborg, Denmark
}

Correspondence should be addressed to A Abood

Email

aliabood8@hotmail.com

\begin{abstract}
Objective: To study pregnancy and pregnancy outcomes in women with primary hyperparathyroidism (PHPT) and in controls. Design: Register-based retrospective cohort study of women aged 16-44 years with a diagnosis of PHPT with age- and gender-matched non-exposed controls in Denmark.

Methods: The patients and controls were identified using the Danish National Hospital Discharge Register for the period 1977-2010. The outcomes were determined using the Birth Register, the Abortion Register and the LPR (National Hospital Discharge Register). The primary outcome was the relative risk of abortions in patients compared with controls.

Results: A total of 1057 women with PHPT and 3171 controls were identified. The number of women giving birth and experiencing abortions did not differ between the two groups (live births, $P=0.21$ and abortions, $P=0.12$ ). Also birth weight, length, Apgar score, and gestation length at abortion did not differ. Within the first year after the diagnosis was made, gestation length was lower in women with PHPT than that in controls. However, this was linked to more deliveries by caesarian sections.

Conclusions: A diagnosis of PHPT did not seem to increase the rate of abortions in our study. Reducing the abortion risk may therefore not be an indication for parathyroidectomy during pregnancy in patients with mild PHPT. The PHPT diagnosis does not seem to affect birth weight, length and Apgar score. The higher number of deliveries by cesarean section after the diagnosis was made may be associated with lower gestation age. The strategy for delivery should be carefully considered in pregnant women with PHPT.
\end{abstract}

\section{Introduction}

Primary hyperparathyroidism (PHPT) ranks third among the most common endocrine disorders after diabetes mellitus and thyroid disease, but it occurs rarely during pregnancy $(1,2,3)$. This can in part be attributed to the fact that PHPT most commonly occurs after the childbearing years. Another explanation could be that the disorder is generally underdiagnosed due to the physiological changes during pregnancy. Hypoalbuminaemia, increased glomerular filtration rate, transplacental transfer of calcium and the increased levels of estrogen all contribute to lowering serum calcium levels, thus masking PHPT (4). In addition, the symptoms that may appear in relation to the disorder, such as nausea and vomiting, may be thought to be due to the pregnancy and not due to PHPT. It is therefore possible that gestational PHPT is more frequent than previously reported.

Although rare, gestational PHPT may have serious consequences for both mother and child $(5,6,7)$. It has been reported that the rate of maternal complications during pregnancy associated with PHPT can be as high as $67 \%$, most commonly being hyperemesis and nephrolithiasis $(4,5)$. More severe complications such as pancreatitis and hypercalcaemic crisis have also been reported, although at extremely high serum calcium levels (6). In addition, an increased risk of preeclampsia has been shown in women with PHPT (8). Gestational PHPT may 
also induce neonatal complications, such as neonatal hypoparathyroidism, hypocalcaemic tetanus, mental retardation and low birth weight $(6,9,10)$.

An association between gestational PHPT and pregnancy loss has been suggested, and so far only one study has been able to determine the rate of pregnancy loss in women with gestational PHPT (5). The study showed a 3.5 higher rate of spontaneous abortions in women with PHPT. The study also showed that surgical intervention in the second trimester might eliminate the observed increased risk of abortion.

The potential consequences of gestational PHPT are numerous, and may be severe. In general, the literature on this subject is limited to case reports $(1,2,3,4,6,7,8,9$, $10,11,12,13,14,15,16,17,18,19,20,21,22)$ and case series $(5,23,24,25,26,27,28)$, probably due to the low incidence. Several of these studies have deemed that surgery in the second trimester is safe for both mother and child, but their findings are based on a small number of patients. Further examination of this disorder is therefore needed.

The aim of this study was to determine the rate of spontaneous abortions and stillbirths in women with PHPT, by examining a larger study population than that reported in the current literature. We also wish to evaluate neonatal outcomes to determine the effects of gestational PHPT on the newborn.

\section{Subjects and methods}

The study was designed as a cohort study, in which exposed subjects were defined as women between 16 and 44 years of age with a diagnosis of PHPT. The PHPT diagnosis was based on the following codes: ICD8 codes: 252.00 (hyperparathyroidismus primarius and adenoma), 252.01 (hyperparathyroidismus primarius and hyperplasia), 252.03 (crisis hyperparathyroidismi), 252.04 (osteitis fibrosa cystica generalisata), 252.05 (Nephrocalcinosis e hyperparathyroidismi), 252.08 (hyperparathyroidismus alia), 252.09 (hyperparathyroidismus) and ICD10 codes: E21.0 (PHPT) and E21.3 (hyperparathyroidism without specification). All patients who were registered in the National Hospital Discharge Register (Sundhedsstyrelsens Landspatientregister, LPR) (29) with a PHPT diagnosis from 1st January 1977 to 31st December 2010 were initially taken into consideration. Among all with a diagnosis of PHPT, women aged 16-44 years were identified, and among these women, pregnancy and outcomes of pregnancy were studied. A total of 1057 women fulfilled the mentioned criteria. For each of these patients, three age- and gender-matched women without PHPT were drawn from the central person register (the national census) from the same period of time.

Information about abortions, live births and stillbirths was obtained through the Abortion Register (from 1977 to 1994 - this register continued from 1995 as a part of LPR, and data from 1995 and onwards were obtained through this register) and the Birth Register (30) (from 1977 to 2010). Furthermore, we were able to obtain serum calcium levels from 41 patients from two of five regions in Denmark.

Primary outcomes were pregnancy, live births, stillbirths and abortions. Secondary outcomes were birth weight, length, Apgar score at $5 \mathrm{~min}$ and length of gestation at abortion. Abortion was defined as termination of pregnancy by the removal or expulsion from the uterus of a foetus or embryo before the stage of viability. An abortion can occur spontaneously, in which case it is usually called a miscarriage, or may be induced for other reasons (e.g. medical reasons). It should be noticed that the Abortion Register was subject to uncertainty during the first years, and that the Birth Register is only updated up to and including the first 6 months of 2010. Based on these parameters, a head-to-head comparison between patients and controls was made.

\section{Statistical analysis}

Mean and s.D.s. were used as descriptive statistics. Comparisons were made using $t$-test for two samples, $\chi^{2}$ test, log-rank test, odds ratio (OR), relative risk (RR) and 95\% CI. To adjust for gestational age, an adjusted SDS for gestational age and gender was calculated.

\section{Results}

A total of 1057 women with PHPT and 3171 controls were identified. The average age among cases was $35.1 \pm 0.2$ years, and was similar in the control group (35.1 \pm 0.1$)$. Table 1 presents the rate of abortions, live births and stillbirths, Apgar score, birth length and birth weight in PHPT patients and controls. In general, no differences were present. Among the PHPT patients, 576 of 1057 (54.5\%) underwent parathyroid surgery during the observation period. In general, it did not change the results presented in Table 1 to limit these to women undergoing surgery for PHPT. The only significant difference was that women who subsequently underwent surgery had more episodes of stillbirth $(9 / 576(1.6 \%)$ vs $1 / 481(0.2 \%)$, $\mathrm{OR}=7.6,95 \% \mathrm{CI} 1.3-44.2, P=0.023)$ before the diagnosis was made. 
Table 1 Births, stillbirths, abortions and Apgar scores in women with primary hyperparathyroidism (PHPT) and controls aged 16-44 years at diagnosis of PHPT.

\section{Variable}

Age at diagnosis (years)

Number of women with at least one live birth before the diagnosis Number of live births before diagnosis

Average length of all live born babies before the diagnosis (SDS)

Average weight of all live born babies before the diagnosis (SDS)

Average Apgar score at 5 min of all live born babies before the diagnosis Number of women with at least one abortion before the diagnosis Pregnancy length at the time of abortion before the diagnosis (weeks) Number of abortions before the diagnosis

Number of women with at least one stillbirth before the diagnosis Number of stillbirths before the diagnosis

Number of women with at least one live birth after the diagnosis

Weight of first live born baby after the diagnosis (SDS)

Length of first live born baby after the diagnosis (SDS)

Average length of all live born babies after the diagnosis (SDS)

Average weight of all live born babies after the diagnosis (SDS)

Average Apgar score at $5 \mathrm{~min}$ of all live born babies after the diagnosis Number of live born babies after the diagnosis

Number of women with at least one abortion after the diagnosis

Pregnancy length at time of abortion after the diagnosis (weeks)

Number of abortions after the diagnosis

Number of women with at least one stillbirth after the diagnosis

Number of stillbirths after the diagnosis

\begin{tabular}{c}
\hline PHPT $(n=1057)$ \\
\hline $35.1 \pm 0.2$ \\
$634(60.0 \%)$ \\
$1.273($ mean 1.2$)$ \\
-0.03 \\
-0.38 \\
$9.9 \pm 0.02$ \\
$220(20.8 \%)$ \\
$9.6 \pm 0.4$ \\
329 \\
$10(0.9 \%)$ \\
10 \\
$179(16.9 \%)$ \\
-0.49 \\
-0.27 \\
-0.27 \\
-0.41 \\
$9.9 \pm 0.03$ \\
262 \\
$58(5.5 \%)$ \\
$8.9 \pm 0.3$ \\
75 \\
$1(0.1 \%)$ \\
1 \\
\end{tabular}

\begin{tabular}{c}
\hline $\boldsymbol{P}$ value \\
\hline- \\
0.17 \\
- \\
- \\
- \\
0.17 \\
0.12 \\
0.62 \\
- \\
0.48 \\
- \\
0.21 \\
- \\
- \\
- \\
- \\
0.13 \\
- \\
0.81 \\
0.85 \\
- \\
0.74 \\
- \\
\hline
\end{tabular}

\section{Pregnancy outcomes}

We stratified time around the time of diagnosis (TOD) to 1-year interval, respectively, before and after TOD (Figs 1, 2,3 and 4). This was done to strengthen the temporal component in a potential causality as much as possible. Periods far from the TOD were divided into 4-year intervals.

Women with PHPT did not experience more abortions than women without PHPT at any time during the observation period (Fig. 1). There was a non-significant excess of abortions in the year following the diagnosis of PHPT among cases, $\mathrm{RR}=1.5 ; 95 \% \mathrm{CI} 0.81-2.74$, and the average gestation age at the time of abortion was around 10 weeks. Women with PHPT had more live births in the year following the diagnosis, $\mathrm{RR}=1.55, P<0.05$ (Fig. 2), than the controls. There was no difference in the rate of stillbirths in the two groups (Table 1). However, women with PHPT who subsequently underwent surgery had a higher rate of stillbirths before the TOD, compared with women with PHPT who did not undergo surgery.

In the year following TOD, a greater number of deliveries by cesarean section were observed among cases (approximately double that of the controls). The proportion of cesarean deliveries among cases remained elevated in the subsequent years after TOD. Gestational length among cases seemed to increase in the subsequent years after TOD, although it remained below the control group. The gestational length was never below 260 days (Figs 3 and 4).

\section{The neonate}

In general, birth weight and birth length of babies born by women with PHPT were similar to the values observed in the control group. There was no difference in Apgar score at $5 \mathrm{~min}$ among cases compared with controls (Table 1).

\section{Discussion}

In this large-scale historic cohort study, few differences in pregnancy-related outcomes compared with controls were present before the diagnosis of PHPT. After the diagnosis was established, more women underwent cesarean delivery - especially within the first year following the diagnosis - probably resulting in reduced gestation length.

The existing literature describing the consequences of gestational PHPT is limited, and mainly consists of case reports or case series $(1,2,3,4,5,6,7,8,9,10,11,12,13$, $14,15,16,17,18,19,20,21,22,23,24,25,26,27,28)$. In total, these studies reported 33 pregnancies in women with PHPT, who did not undergo surgery. Among these 33 pregnancies, 19 (58\%) resulted in uneventful deliveries, 

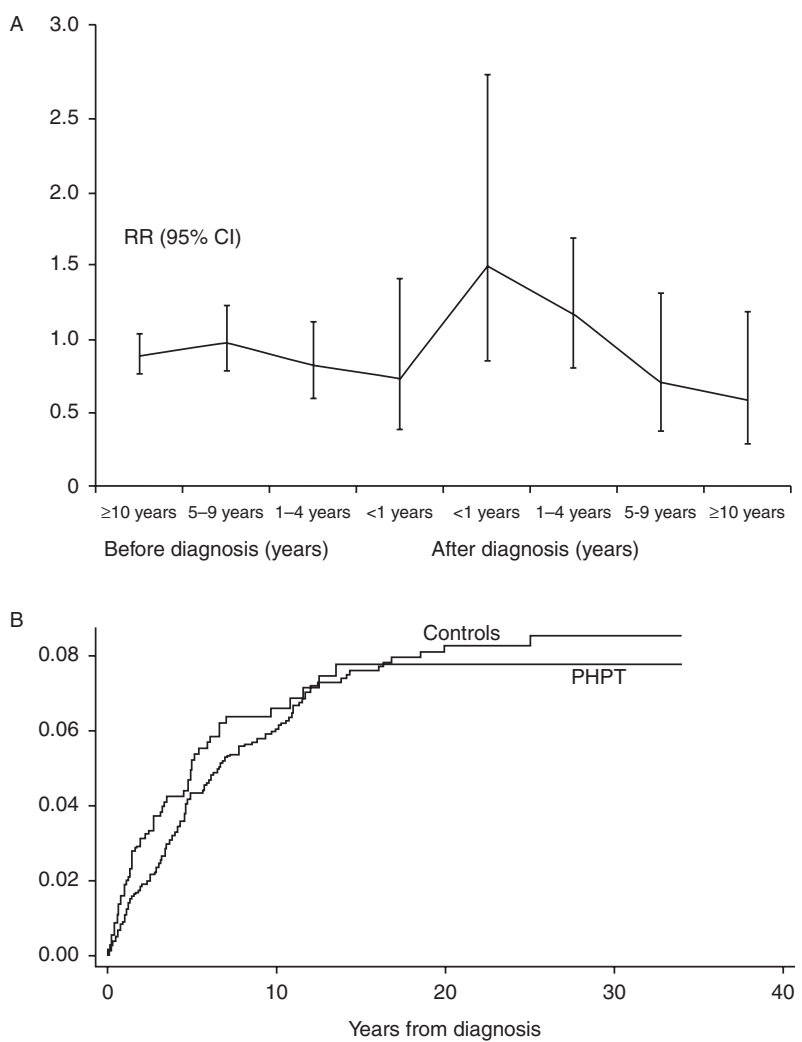

Figure 1

Risk of abortions in women with primary hyperparathyroidism (PHPT). Women aged 16-44 years at diagnosis of PHPT having an abortion before and after the diagnosis was made.

(A) Relative risk of abortions in patients compared with controls stratified by time before and after diagnosis was made. (B) Time until first abortion after diagnosis as cumulated hazard plot (Nelson-Aalen cumulative hazard estimates), $P=0.59$ by the log-rank test.

eight (24\%) ended in cesarean delivery and six $(18 \%)$ in abortions or pre-term deliveries. In the analysis of these case reports, it should be observed that women with health problems in pregnancy, such as impaired growth of the foetus or pregnancy loss, may be more likely to be screened, and thus have their calcium levels measured and therefore have a diagnosis of an otherwise undetected PHPT. This would led to a higher catchment of PHPT in pregnancies with adverse outcomes. A biased reporting may thus have taken place, i.e. only the more severe cases may be reported and especially those resulting in abortions and pre-term deliveries.

The number of pregnancies was thus much larger in our study. We were not able to prove a statistically significant association between gestational PHPT and the rate of abortions in the hitherto largest study of its kind. A total of 1057 women with PHPT had their obstetrical history reviewed, showing no increased risk of abortions in patients with PHPT. The 3.5-fold increase in the rate of abortions among women with PHPT shown in the study by Norman et al. (5) could thus not be confirmed through the findings of our study. Although the results of our study were apparently in contrast to those of Norman et al. (5), it should be underlined that they are not completely comparable. First of all, in the study by Norman et al. (5), $87 \%$ of the pregnancies took place in females with a maternal serum calcium level of $10.8 \mathrm{mg} / \mathrm{dl}$ (albumin adjusted levels $2.7 \mathrm{mmol} / \mathrm{l}$ and ionised calcium $1.47 \mathrm{mmol} / \mathrm{l}$ ) or above. Second, Norman et al. (5) consider all pregnancies in a 5-year period before the TOD as being representative of a potential association between PHPT

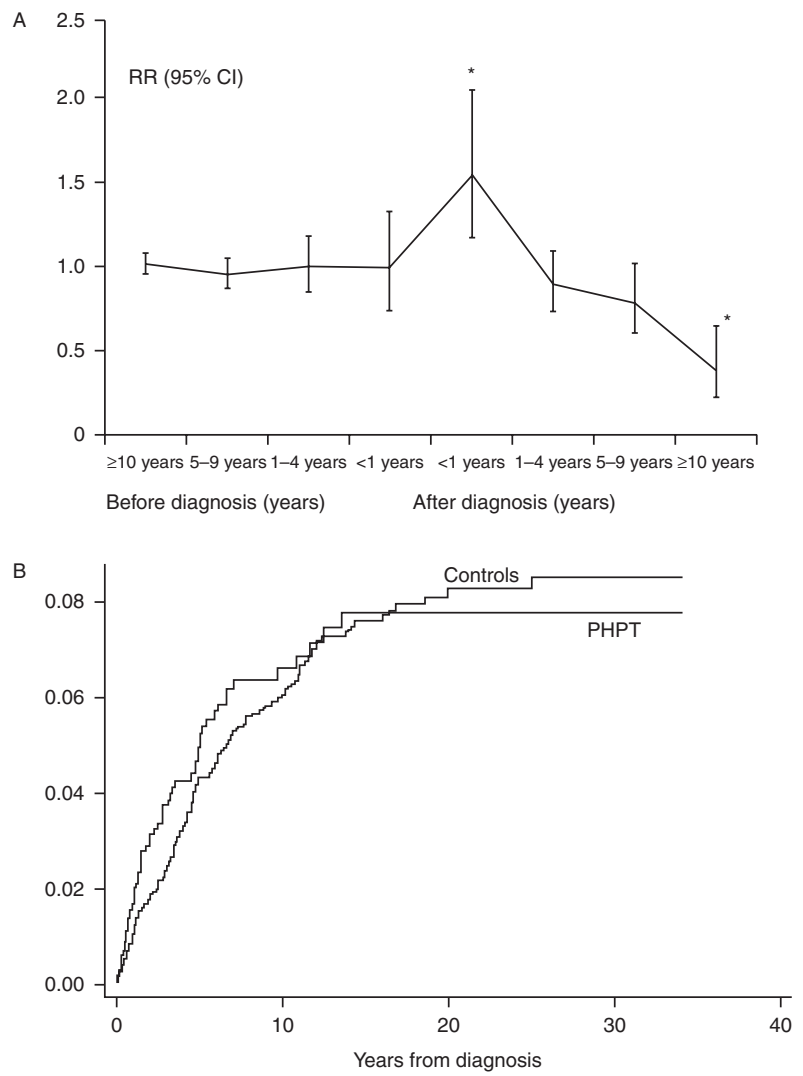

\section{Figure 2}

Occurrence of live births. Women aged 16-44 years at diagnosis giving birth. (A) Relative occurrence of live births in patients compared with controls before and after the diagnosis was made, ${ }^{*} P<0.05$. (B) Time until first live birth after diagnosis as cumulated hazard plot (Nelson-Aalen cumulative hazard estimates), $P=0.50$ by the log-rank test. 


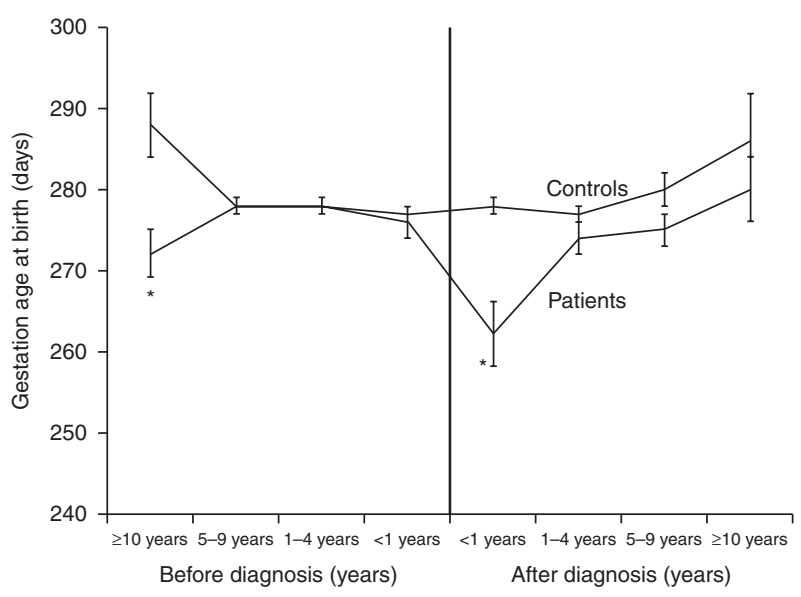

\section{Figure 3}

Gestation age at birth before and after diagnosis in patients and controls. ${ }^{*} P<0.05$ by $y$-test for two samples. Gestation age at birth (days) - note only data from 1997 and onwards.

and pregnancy loss. In addition, Norman et al. (5) did not include a control group for comparison when the 3.5-fold increased risk of abortions was found. The rate was determined by comparing their results to the abortion rate in the general population. Third, the number of women with PHPT evaluated in our study $(n=1057)$ exceeds by far the 32 women in the study by Norman et al. (5), which is a clear strength of our study. Our population represents a broad spectrum of patients with PHPT - mildly as well as severely affected. We therefore cannot rule out that patients with severe PHPT may experience an increase in the rate of abortions. Our study shows that the PHPT diagnosis itself is not associated with an increased risk of abortion. In our study, we stratified time around the TOD, so that we looked at RRs for abortions within 1 year before diagnosis and within 1 year after diagnosis respectively. These smaller time intervals used in our study are more likely to be representative of a causal association between gestational PHPT and the rate of abortions. Furthermore, we made a head-to-head comparison with healthy pregnant women without PHPT to illustrate the difference in the rate of abortions between the two groups, for which Norman et al. (5) used the abortion rate in the general population.

Our study also shows that women with PHPT had more live births in the year following the diagnosis when compared with controls. It is doubtful whether this is caused by PHPT. It is more likely the result of a surveillance bias - pregnant women being more likely to have serum calcium measured, i.e. the diagnosis is made during pregnancy leading to more women giving birth in the year following the diagnosis of PHPT. This may also be the reason why the birth rate declines later. The diagnosis is made, and the woman may not wish further pregnancies as she has had the number of children she wants.

The difference in stillbirth before the diagnosis of PHPT later requiring surgery may suggest that these women had more severe disease. However, we were able to obtain serum calcium levels on 41 patients from two of five regions in Denmark, among whom no differences in plasma calcium levels were present. Twelve of these patients had experienced one or more pregnancy losses before diagnosis, while 29 had not experienced any pregnancy loss before diagnosis. A comparison of mean ion-calcium levels in the two patient groups shows that they were statistically identical $(P=0.20)$. There were 12 women with no history of live births before diagnosis, and they had a significantly elevated mean ion-calcium level of $1.52 \pm 0.14, P=0.03$, when compared with the 29 women who did have a history of live births before diagnosis. More extensive information about serum calcium levels is thus needed to estimate disease severity, as serum calcium levels in these patients, who most often have equilibrium hypercalcaemia, do not always per se reflect the severity of disease. The rate of change in serum calcium levels may of greater importance, as these fluctuations may be more decisive in the development of complications such as renal affection.

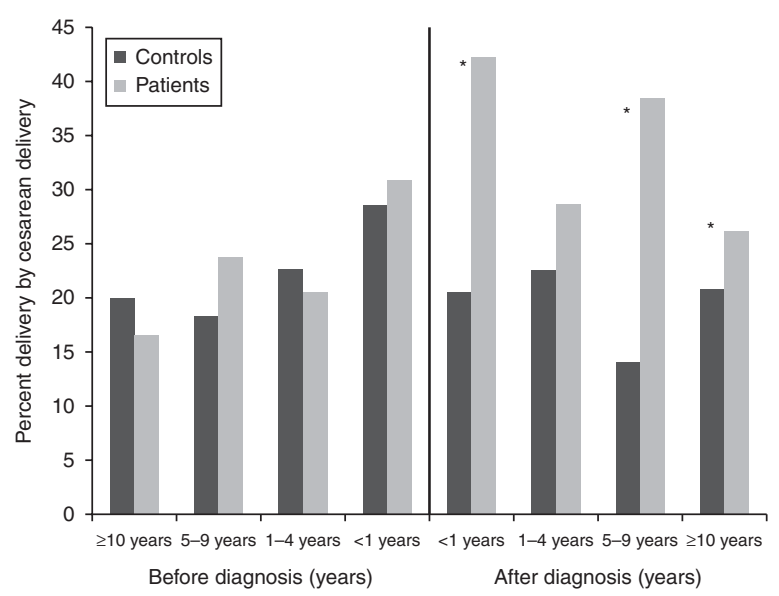

\section{Figure 4}

Cesarean delivery. Proportion of patients and controls delivering by cesarean section before and after the diagnosis. $\star P<0.05$ by $\chi^{2}$-test. 
The length and weight of live born babies were statistically identical when comparing cases with controls. During the first year after diagnosis, gestation length was reduced among cases. However, it should be noticed that more cesarean deliveries took place in the same period of time, probably explaining the reduction in gestation length. After the first year after diagnosis, gestation length increased, but more babies were still delivered by cesarean section. This trend towards cesarean delivery may be due to the fact that after one cesarean section, babies are more likely to be delivered by cesarean section. However, this does not explain the dramatic increase in the proportion of cesarean delivery among cases during the first year after TOD. We therefore cannot exclude that PHPT may increase the risk of delivering by cesarean section. In our data, we did not have access to whether the reason was emergency or elective caesarean delivery, which is a weakness. However, in our opinion, the main figure is the overall rate, as most caesarian deliveries would be planned. Owing to the underlying condition, some departments may opt for elective caesarean delivery.

Although the gestational length is reduced, it does not result in preterm births, as it remains above 259 days (37 weeks), which is the limit for preterm delivery. Also average birth weight seems to remain in the normal zone, and the Apgar score is unaffected as well. It is thus possible that gestational PHPT may increase the risk of delivering by cesarean delivery, although it does not result in preterm delivery and does not seem to compromise the neonate in terms of being underweight. Instead, the maternal PHPT may cause suppression of the parathyroid glands in the neonate, resulting in neonatal hypocalcaemia. In the studies reviewed, neonatal hypocalcaemia was observed in $\sim 50 \%(1,2,3,4,5,6,7,8,9,10,11,12,13,14,15,16,17$, $18,19,20,21,22,23,24,25,26,27,28)$. It should be noticed that many of these patients did not receive medical treatment during the pregnancy, as PHPT was diagnosed afterwards. Several studies have reported the absence of neonatal hypocalcaemia when the pregnant women are treated conservatively during the pregnancy. Furthermore, it was possible to correct hypocalcaemia in the newborn through calcium infusions in almost every case, and without complications for the newborn.

Several studies have deemed surgery in the second trimester as safe and of benefit for the pregnant women $(5,6,7,16,24,26)$. In the study by Norman et al. (5), most pregnancies were lost before or in week 13 (21/30 - 70\%), i.e. before the second trimester, and as surgery was performed in the second trimester not all pregnancy losses could be prevented. Our study illustrates the same tendency of abortions occurring before the second trimester as the average week of abortion was around week 10 . The lower week of abortion in our study was due to induced abortions (abortus provocatus), which by law cannot be performed later than week 12. From our study, it is thus questionable if surgery in the second trimester (weeks 14-26) would prevent many abortions in a broad spectrum of PHPT patients, especially when the PHPT diagnosis itself does not seem to be a risk factor for abortions. However, we are not able to make any statements regarding the risk of abortions in severely affected patients, as this would require a serum calcium stratification in the 1057 women. It was unfortunately not possible to obtain such data. Parathyroidectomy in the second trimester might therefore still be of benefit for women with severe PHPT, as stated in the current literature. Some would say that the purpose of surgery is to prevent neonatal hypocalcaemia, which indeed can be achieved by surgery. But as the condition can be successfully corrected by medical treatment and without serious consequences for the newborn, it should be held up against the risk in relation to surgery (infection, complications to anesthesia, etc.). The strategy should thus be considered carefully.

There is a large spectrum of PHPT and it is highly likely that severe symptomatic disease has different maternofoetal effects compared with mild disease. Women requiring surgery have more advanced disease than those not requiring surgery. In our study, no differences were present between those undergoing surgery and those not undergoing surgery, which may corroborate the absence of a detrimental effect of PHPT on pregnancy outcomes. Among the patients whose biochemical data were available, the level of plasma calcium was in the range for which surgery is advocated in non-pregnant patients according to the international guidelines, which are also followed in Denmark $(31,32)$.

The major limitations of our study are the retrospective design and the fact that it is register based. A further weakness is the lack of information on the rate of neonatal hypocalcaemia. However, infant outcomes evaluated by other measures seemed identical. As it was not possible to obtain serum calcium, PTH and vitamin D levels from the 1057 women with a diagnosis of PHPT, the specific criteria for diagnosis in each case are unknown, which is another limitation of our study. In general, the validity of the codes is high in the National Hospital Discharge Register $(29,30)$. The diagnosis of PHPT is rigorous and based on measurements of plasma PTH, plasma calcium, urinary calcium, plasma phosphate 
levels, and for localisation diagnosis sestamibi scintigraphy (although this is not possible in pregnancy) and ultrasound of the parathyroids. In some cases, genetic testing is carried out to eliminate familial hypocalciuric hypercalcaemia. Also, among the 41 patients whose serum calcium levels were available, the diagnosis was confirmed in all, therefore in general misclassification seems limited.

\section{Conclusion}

In conclusion, a diagnosis of PHPT does not seem to be associated with an increased risk of abortions, but might be associated with an increased risk of delivering by cesarean section. As our results are based on a broad spectrum of PHPT patients, we conclude that mildly affected patients do not have an increased risk of abortions. Parathyroidectomy in the second trimester may thus not reduce the risk of abortions in these patients. However, it is uncertain whether severe PHPT could increase the risk of abortions. Parathyroidectomy in the second trimester might therefore still be of benefit for severely affected patients, although this requires further research.

\section{Declaration of interest}

The authors declare that there is no conflict of interest that could be perceived as prejudicing the impartiality of the research reported.

\section{Funding}

This research did not receive any specific grant from any funding agency in the public, commercial or not-for-profit sector.

\section{References}

1 Iqbal N, Aldasouqi S, Peacock M, Mohammed IA \& Edmondson JW. Life-threatening hypercalcemia associated with primary hyperparathyroidism during pregnancy: case report and review of literature. Endocrine Practice 19995 337-342. (doi:10.4158/EP.5.6.337)

2 Harsoulis F, Karayiannis B, Karvounaris D \& Mamopoulos M. Primary hyperparathyroidism in pregnancy. Journal of Obstetrics and Gynaecology 200020 188-189. (doi:10.1080/01443610063039)

3 Cherry TA, Kauffman RP \& Myles TD. Primary hyperparathyroidism, hypercalcemic crisis and subsequent seizures occurring during pregnancy: a case report. Journal of Maternal-Fetal \& Neonatal Medicine 2002 12 349-352. (doi:10.1080/jmf.12.5.349.352)

4 Som M \& Stroup JS. Primary hyperparathyroidism and pregnancy. Proceedings 201124 220-223.

5 Norman J, Politz D \& Politz L. Hyperparathyroidism during pregnancy and the effect of rising calcium on pregnancy loss: a call for earlier intervention. Clinical Endocrinology 200971 104-109. (doi:10.1111/ j.1365-2265.2008.03495.x)

6 Amaya Garcia M, Acosta Feria M, Soto Moreno A, Dios Fuentes E, Navarro González E, Quijada Thong D, Del Valle A, Acosta Delgado D \& Astorga Jiménez R. Primary hyperparathyroidism in pregnancy.
Gynecological Endocrinology 200419 111-114. (doi:10.1080/ 09513590400002334)

7 Jesudason WV, Murphy J \& England RJ. Primary hyperparathyroidism in pregnancy. Journal of Laryngology and Otology 2004118 891-892. (doi:10.1258/0022215042703714)

8 Schnatz PF \& Thaxton S. Parathyroidectomy in the third trimester of pregnancy. Obstetrical \& Gynecological Survey $200560672-682$. (doi:10.1097/01.ogx.0000180889.23678.fb)

9 McDonnell CM \& Zacharian MR. Maternal primary hyperparathyroidism: discordant outcomes in a twin pregnancy. Journal of Paediatrics and Child Health 200642 70-71. (doi:10.1111/j.1440-1754.2006.00790.x)

10 Ip P. Neonatal convulsion revealing maternal hyperparathyroidism: an unusual case of late neonatal hypoparathyroidism. Archives of Gynecology and Obstetrics 2003268 227-229. (doi:10.1007/ s00404-002-0316-5)

11 Petousis S, Kourtis A, Anastasilakis CD, Makedou K, Giomisi A, Kalogiannidis I, Margioula-Siarkou C, Xanthopoulou E \& Rousso D. Successful surgical treatment of primary hyperparathyroidism during the third trimester of pregnancy. Journal of Musculoskeletal \& Neuronal Interactions 201212 43-4.

12 Malekar-Raikar S \& Sinnott BP. Primary hyperparathyroidism in pregnancy - a rare cause of life-threatening hypercalcemia: case report and literature review. Case Reports in Endocrinology 2011. Article ID 520516. (doi:10.1155/2011/520516)

13 Kokrdova Z. Pregnancy and primary hyperparathyroidism. Journal of Obstetrics and Gynaecology 201030 57-59. (doi:10.3109/ 01443610903315611)

14 Krysiak R, Wilk M \& Okopien B. Recurrent pancreatitis induced by hyperparathyroidism in pregnancy. Archives of Gynecology and Obstetrics 2011284 531-534. (doi:10.1007/s00404-010-1668-x)

15 Perin E, Cacciaguerra G, Lapenna R, Catena C, Sechi LA \& Marchesoni D. Primary hyperparathyroidism in pregnancy. Fertility and Sterility 200890 2014.e13-2014.e15.

16 Tollin SR. Course and outcome of pregnancy in a patient with mild, asymptomatic, primary hyperparathyroidism diagnosed before conception. American Journal of the Medical Sciences 2000320 144-147. (doi:10.1097/00000441-200008000-00014)

17 Morton A \& Pyke C. Anaemia and hyperparathyroidism in pregnancy. ANZ Journal of Surgery 200575 253-254. (doi:10.1111/j.1445-2197. 2005.03344.x)

18 Haenel LC 4th \& Mayfield RK. Primary hyperparathyroidism in a twin pregnancy and review of fetal/maternal calcium homeostasis. American Journal of the Medical Sciences 2000319 191-194. (doi:10.1097/ 00000441-200003000-00011)

19 Carella MJ \& Gossain VV. Hyperparathyroidism and pregnancy: case report and review. Journal of General Internal Medicine 19927 448-53. (doi:10.1007/BF02599166)

20 Lueg MC \& Dawkins WE. Primary hyperparathyroidism and pregnancy. Southern Medical Journal 198376 1389-1392. (doi:10.1097/ 00007611-198311000-00017)

21 Kirby CA \& Read MD. Primary hyperparathyroidism in pregnancy. Australian \& New Zealand Journal of Obstetrics \& Gynaecology 198222 121-122. (doi:10.1111/j.1479-828X.1982.tb01421.x)

22 Zanoner C. Case of habitual abortion in a patient with primary hyperparathyroidism (current obstetrical clinico-diagnosticotherapeutic and statistical aspects). Il Friuli Medico 196924 227-240.

23 McMullen TP, Learoyd DL, Williams DC, Sywak MS, Sidhu SB \& Delbrige LW. Hyperparathyroidism in pregnancy: options for localization and surgical therapy. World Journal of Surgery 201034 1811-1816. (doi:10.1007/s00268-010-0569-2)

24 Truong MT, Lalakea ML, Robbins P \& Friduss M. Primary hyperparathyroidism in pregnancy: a case series and review. Laryngoscope 2008118 1966-1969. (doi:10.1097/MLG.0b013e318180276f)

25 Hsieh YY, Chang CC, Tsai HD, Yang TC, Chiu TH \& Tsai CH. Primary hyperparathyroidism in pregnancy - report of 3 cases. Archives of Gynecology 1998261 209-214. (doi:10.1007/s004040050223) 
26 Gidiri M, Lindow SW, Masso EA \& Philips K. Parathyroidectomy in pregnancy for primary hyperparathyroidism with successful pregnancy outcome: a report of two pregnancies. Journal of Obstetrics and Gynaecology 200424 318-319. (doi:10.1080/ 01443610410001661011)

27 Gelister JS, Sanderson JD, Chapple CR, O'Riordan JL, Cowie AG \& Milroy EJ. Management of hyperparathyroidism in pregnancy. British Journal of Surgery 198976 1207-1208. (doi:10.1002/bjs. 1800761128)

28 Montoro MN, Collea JV \& Mestman JH. Management of hyperparathyroidism in pregnancy with oral phosphate therapy. Obstetrics and Gynecology 198055 431-434.
29 Andersen TF, Madsen M, Jørgensen J, Mellemkjoer L \& Olsen JH. The Danish National Hospital Register. Danish Medical Bulletin 1999 46 263-268.

30 The Danish Birth Register, http://www.ssi.dk/Sundhedsdataogit/ Registre.aspx (accessed 11/1-2013).

31 Proceedings of the NIH Consensus Development Conference on diagnosis and management of asymptomatic primary hyperparathyroidism. Bethesda, Maryland, October 29-31, 1990. Journal of Bone and Mineral Research 19916 S1-S166. (doi:10.1002/jbmr.5650061302)

32 Bilezikian JP, Khan AA \& Potts JT Jr. Guidelines for the management of asymptomatic primary hyperparathyroidism: summary statement from the Third International Workshop. Journal of Clinical Endocrinology and Metabolism 200994 335-339. (doi:10.1210/jc.2008-1763)

Received 27 November 2013

Revised version received 12 April 2014

Accepted 16 April 2014 